\title{
Review and Proposal of Alternative Technologies for Comprehensive and Reliable Newborn Screening Using Paper Borne Urine Samples for Lysosomal Storage Disorders: Glycosphingolipid Disorders \\ Journal of Inborn Errors of Metabolism \& Screening 2021, Volume 9: e20200011 DOI: https://doi.org/10.1590/2326-4594- JIEMS-2020-0011
}

\author{
José Ramón Alonso-Fernández ${ }^{1,2}$ (1) and Javier Fidalgo López ${ }^{3}$ (1)
}

\begin{abstract}
Few current methods are efficient to detect a high number of lysosomal storage disorders (LSDs) in newborn screening. Therefore, we propose a stepwise procedure that starts with the use of paper borne urine samples (Berry-Woolf specimen) for the inexpensive detection of elevated lysosomal content and the identification of which of the three majors biochemical groups mucopolysaccharides, oligosaccharides, and glycosphingolipids - is detected. Urine samples are preferable to blood samples because of their higher concentrations of the relevant analytes. Subsequent steps would precisely determine which enzyme deficiency is involved. As a summary, following our previous papers on the detection of elevated oligosaccharides and mucopolysaccharides, here we describe how elevated urinary glycosphingolipids (GSLs) could be fluorometrically detected using the reagent 5-hydroxy1-tetralone (HOT) and subsequently identified with precision by continuous thin layer chromatography or other techniques. We also outline the steps required for the validation of this procedure for its introduction in newborn screening programs.
\end{abstract}

Keywords: Newborn screening, lysosomal storage disorders (LSDs), urine samples impregnated in paper, Berry-Woolf specimen, glycosphingolipids.

\section{Introduction}

Lysosomal storage disorders (LSDs) comprise a heterogeneous group of approximately seventy [1] inborn errors of metabolism (IEMs) caused by the absence or malfunction of hydrolases required to degrade certain complex substrates into simpler molecules inside cellular lysosomes. Owing to this enzymatic malfunction, the undegraded substrates (lipids, sulfates, complex carbohydrates, etc.) accumulate inside certain cell types, which can affect multiple organs and tissues [2]. The majority of these disorders are typically inherited in an autosomal recessive manner, many of them resulting from the mutation of a gene encoding for an essential lysosomal enzyme. Although the isolated prevalence of each disorder is low, the joint prevalence of LSDs at birth has been reported as around 1 in 7,500 [3, 4].

According to the nature of the abnormal accumulation of lysosomal contents, LSDs can be classified as mucopolysaccharidoses, GM2 ganglisidoses, glycoproteinoses, neutral glycosphingolipidoses, mucolipidoses, leukodystrophies, glycogen storage diseases, disorders of neutral lipids, or disorders of protein transport or trafficking [5]; other more detailed classifications can be made [1]. Alternatively, just three major groups can be distinguished, mucopolysaccharidoses, oligosaccharidoses, and glycosphingolipidoses.

Since some LSDs can now be treated (e.g., by means of stem cell therapy, enzyme replacement therapy, substrate reduction therapy, or bone marrow transplantation [6] or with CRISPR/ Cas9 genome editing as a promising further possibility $[7,8]$ and other genetic and pharmacologically chaperone therapies [1]),

\footnotetext{
1 Universidade de Santiago de Compostela, Departamento de Pediatría, Santiago de Compostela, Galicia, Spain.

${ }^{2}$ Complexo Hospitalario Universitario de Santiago - CHUS-SERGAS, Hospital Clínico, Laboratorio de Peneirado Neonatal en Galicia, Laboratorio de Metabolopatías, Santiago de Compostela, Galicia, Spain. ${ }^{3}$ Independent research, Vigo, Galicia, Spain.
}

Received August 12, 2020, and in revised form March 17, 2021. Accepted for publication March 29, 2021.

Corresponding Author:

José Ramón Alonso-Fernández, Email: joseramon.alonso@usc.es 
calls for their early detection in newborn screening programs have been increasing, with a view to preventing the accumulation of pathogenic substrates through the early initiation of treatment [9].

However, current methods for LSD detection are ill-suited to newborn screening as they are costly and have a limited or nonexistent multiplexing capacity [10]. A more cost-efficient procedure, preferably one that fits easily into existing newborn screening programs so as to take maximum advantage of the existing relevant infrastructure, is urgently needed.

Most current newborn screening programs are based on the use of paper borne heel prick blood samples introduced by Guthrie in the early days of screening for phenylketonuria (PKU). Consequently, most LSD detection methods have likewise been designed for dried blood samples. They include the fluorometric analysis of enzymatic activity developed by Chamoles for $\alpha$-L-iduronidase (deficient in type I mucopolysaccharidosis) [11], $\alpha$-galactosidase (deficient in Fabry disease) [12], and $\beta$-Dgalactosidase (deficient in GM1 gangliosidosis) [13] as well as immunochemical methods targeting lysosomal proteins [14] and the determination of enzymatic activity deficit by tandem mass spectrometry (MS/MS) [5, 9, 15-19]. However, the dependence of these methods on dried blood samples is misguided because LSD relevant analytes are excreted in urine at concentrations several times higher than those reached in blood [20], as stressed by authors who have used both matrices $[14,15]$ and have accordingly criticized the absence of urine samples in newborn screening programs. Furthermore, blood spot-based enzymatic analyses require a separate disk of blood impregnated filter paper for each LSD.

The absence of urine samples in newborn screening programs is widespread but not universal. Urine was the matrix employed in the original newborn screening programs [20], and paper-borne urine samples (Berry-Woolf specimens) are still used in our, laboratory to screen for amino acids disorders, sugar disorders [21], and in recent years, two groups of LSD: mucopolysaccharidoses and oligosaccharidosis. Their collection and use are at most, marginally more demanding than those of blood spots [22], and their introduction in screening programs that are currently and exclusively blood spot based would not be difficult. It may be noted that 21 hydroxylase deficiency (the major form of congenital adrenal hyperplasia) is another IEM that is more efficiently screened for in urine than in blood [22].

The procedures we employ in screening for mucopolysaccharidoses and oligosaccharidosis [23-28] form part of a general efficient screening strategy for LSDs that combines the above-noted advantages of urine samples with the specificity of other methods for individual LSDs [29]. In the first instance, we apply inexpensive, high throughput absorptiometric methods to Berry-Woolf specimens to detect any elevation of relevant analytes and identify whether the elevated substances are mucopolysaccharides or oligosaccharides; these results are obtained on the day of sample receipt. Positive tests at this stage prompt the precise identification of the elevated metabolites by continuous thin layer chromatography (cTLC) [25-31] (though in view of the low number of false positives [FPs], other methods could be used at this stage with little effect on overall efficiency). When desirable, the genetic alteration is identified by DNA sequencing. Then inexpensive, high throughput, sensitivity oriented first stage analysis follows only when necessary by procedures that can identify specific LSDs; this approach contrasts those of authors who, while supporting the use of urine samples, use them only to identify a single disease [32].

In this paper, we describe how the above strategy can be extended to the third of the three major groups of LSDs, glycosphingolipidoses. For the first stage (the detection of elevated generic glycosphingolipids), we propose adapting the technique developed by Watanabe et al. [33], which is based on the fluorometric determination of the complex formed in the presence of sulfuric acid by the reaction between glycosphingolipids and 5-hydroxy-1-tetralone(HOT) (Figure 1).

\section{CAS Registry Number 28315-93-7 $\quad \mathbf{C}_{10} \mathbf{H}_{10} \mathbf{O}_{2}$}

1(2H)-Naphthalenone, 3,4-dihydro-5-hydroxyOther Names

- 3,4-Dihydro-5-hydroxy-1(2H)-naphthalenone

- 1,2,3,4-Tetrahydro-1-oxo-5-hydroxynaphthalene

- 1,2,3,4-Tetrahydro-5-hydroxynaphthyl-1-one

- 5-Hydroxy-1,2,3,4-tetrahydronaphthalen-1-one

- 5-Hydroxy-1-tetralone

- 5-Hydroxy-3,4-dihydro-1(2H)-naphthalenone

- 5-Hydroxy-3,4-dihydro-2H-naphthalen-1-one

- 5-Hydroxy-a-tetralone

- 5-Hydroxytetralone

- HOT

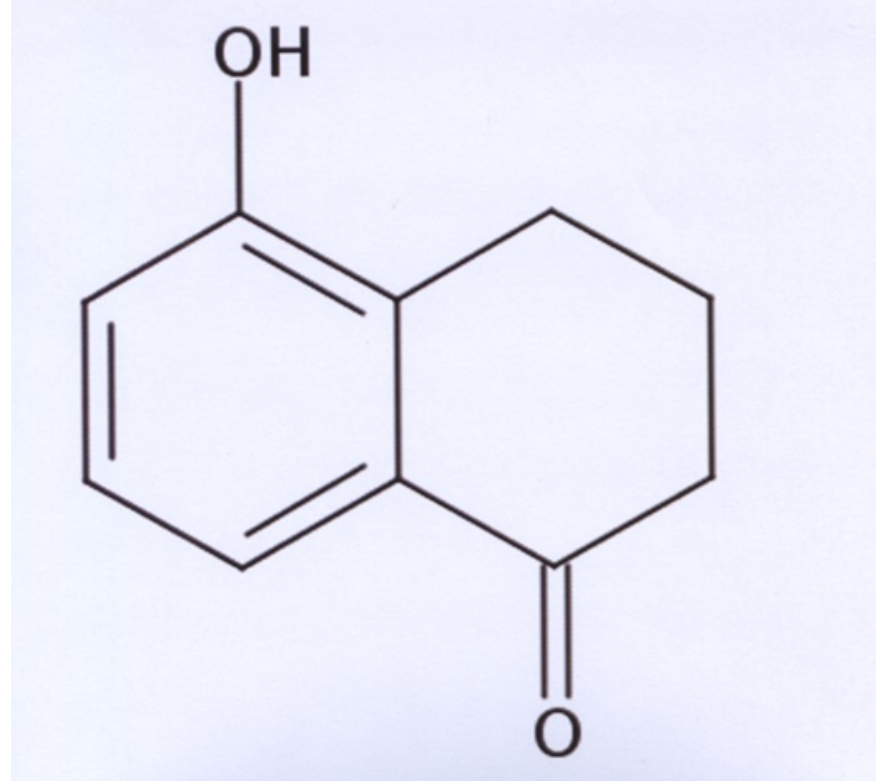

Figure 1. HOT 
As far as we know, this method has never been implemented in 96 well microtiter plates for high throughput newborn screening. Following a description of the proposed adaptation, we indicate possibilities for the second (identification) stage of the procedure, outline the necessary validation procedures for the effective introduction of this strategy in newborn screening programs, and conclude with a number of general remarks.

\section{Urine Specimen Collection and Elution}

In our screening program, Berry-Woolf specimens are taken from newborns simultaneously with blood samples (Guthrie specimens) on the third day of life [34]. Each urine sample (generally, as a result of reflex urination upon heel prick) is impregnated in a slip of Whatman 903 or Munktell TFN sorbent paper that is included in the sample collection kit issued for each newborn in Galicia (NW Spain) [22].

Once the urine samples arrive at the laboratory (by hand or via ordinary mail), eluates are obtained from each sample by stirring four disks $6 \mathrm{~mm}$ in diameter (or sixteen disks $3 \mathrm{~mm}$ in diameter) in one well of a 96 well microtiter plate containing 300 $\mu \mathrm{L}$ of ultrapure water per well. Transferring glycosphingolipids from the paper borne urinary sediment to the eluate requires a 10-minute ultrasound application (e.g., by means of a 96-probe ultrasound device).

\section{Determination of Glycosphingolipids}

Watanabe et al.'s [33] method for determining glycosphingolipids can be adapted for newborn screening essentially as follows':

- Transfer $20 \mu \mathrm{L}$ of the urine eluate to an acid resistant black quartz microtiter plate.

- Mix the eluate with $200 \mu \mathrm{L}$ of the HOT reagent (0.1\% HOT in $80 \% \mathrm{H} 2 \mathrm{SO} 4$ solution) for $10 \mathrm{~min}$ at $120^{\circ} \mathrm{C}$.

- Cool the reaction mixture to $0^{\circ} \mathrm{C}$ and measure the fluorescence with excitation and emission wavelengths of 475 and $530 \mathrm{~nm}$, respectively.

However, optimization will no doubt alter the details of this procedure, such as the excitation and emission wavelengths used for fluorometry. Furthermore, since the HOT reagent also reacts with saccharides and oligosaccharides, calibrating with glucose and subtracting the concentration of oligosaccharides in the eluate will be necessary (oligosaccharides can be determined using an anthrone reagent that does not react with glycosphingolipids [25]).

1 In this concept paper, we do not disclose our detailed recipe, hoping that others devised by those interested in this proposal will emerge, which will most probably require modifications with regard to putting them into routine practice. The specimen quantity and elution volume are those being used today in the laboratory in Galicia for other detection methods sufficient to extend to these and other screening programs.
Thus, oligosaccharides and glycosphingolipids can be determined successively in the same eluate. Finally, normalizing the measured glycosphingolipid concentration with respect to creatinine, which can be measured by the modified Jaffe method currently employed in us laboratory [23-25], will be necessary. This standardization ensures that the variation in analyte concentration from the amount of liquid intake does not influence the results.

\section{Identification of Elevated Glycosphingolipids}

The elevated glycosphingolipids $[35,36]$ can be identified by cTLC. A few necessary cases will depend on the cut of which is established. In any case, in our simple procedure, the urine specimens are analyzed nine at a time. This technique has many variations, some old and outdated. Ours [25-31] uses the same reagent as before (HOT) [33] and authentic glycosphingolipid samples as references with which to compare the retention factor(s) of the sample. If the urine sample must be concentrated or diluted, this can be done as described in a paper $[28]^{2}$. However, since at this stage, high throughput is not needed, other analytical methods could also be employed, such as blood spot-based fluorometric analysis, liquid chromatography-tandem mass spectrometry (LC-MS/MS), or nuclear magnetic resonance (NMR) techniques. The genetic alteration responsible for the elevated glycosphingolipid levels can be identified by DNA sequencing $[37,38]$.

\section{Evaluation, Optimization for Newborn Screening, and Validation}

Once optimized, the performance of the method proposed for the first stage detection of elevated glycosphingolipids must be characterized by determining its precision (within and between run coefficients of variation), accuracy, recovery, linearity, sensitivity, an vulnerability to interference. The potential utility of the overall two stage procedure for newborn screening must then be evaluated by determining its diagnostic sensitivity and specificity, positive and negative predictive values, positive and negative likelihood ratios, and diagnostic efficiency using a cutoff obtained from a receiver operating characteristic (ROC) curve constructed with data from a suitable sample (though the use of ROC curves for evaluating newborn screening results is questionable [39]). Once a suitably large real series of newborns has been screened (e.g., 2,000), a more appropriate cutoff can be established (e.g., 90th or 95th percentile) and the diagnostic quality parameters recalculated.

2 This concentration method is ten times faster and more practical than impregnating and drying the paper ten times or multiplying the liquid urine depositions on the chromatographic plate by 10 and drying between each deposition. 


\section{Concluding Remarks}

The LSD screening strategy proposed in this and previous papers is unconventional in two respects. First, it proposes resuming the collection of dried paper borne urine samples (a procedure never abandoned by our lab). As previously noted, urine is preferable to blood for the detection of LSDs (also for congenital adrenal hyperplasia) because it has higher concentrations of the relevant analytes, whereas the sensitivity and specificity of blood spot-based methods are questionable [40-46]. However, most of the literature on newborn screening for LSDs (La Marca's review [47] is an exception) assumes that the sample must be a blood spot. Conversely, as we have already stated, dry urine samples can be collected reliably and easily at a minimal additional cost, most probably far less than that incurred as a result of the relatively poor specificity of many blood spot-based methods, owing to the low concentrations of analytes in blood spots. Furthermore, for analysis based on the determination of enzymatic activity, blood spots also have a disadvantage in that the relevant enzymes are degraded by temperatures higher than approximately $24^{\circ} \mathrm{C}$ [48], possibly caused by their adherence to the fibers of the filter paper $[49,50]$ (which can also affect the epitopes on which immunochemical methods rely $[49,50])$. These problems do not arise when determining the targeted analytes by us methods. No scientifically or economically valid reason exists for not using paper borne urine samples to screen for disorders in which the appropriate analytes are more abundant in urine than in blood.

Second, our proposal is based on relatively unsophisticated analytical technology and, if validated, would make LSD screening available to communities that cannot afford high tech approaches. For a laboratory such as ours that already possesses an MS/MS apparatus (for example), its use would be reserved for the second LSD identification stage, thus reducing costs and freeing the apparatus for other tasks. Perhaps even more important for such a laboratory is the relative simplicity of our methods, prone to producing fewer issues than complex methods.

Finally, agencies still oppose LSD screening on the grounds that our understanding of these disorders is insufficient and that existing screening methodologies are unproven. Is it necessary to insist that the worth of a methodology cannot be proven if it has not been tried and that the same goes for therapies now available? A paper review lysosomal storage disease [51]. A couple of 2018 papers that had dealt with reviews of the issue $[52,53]$ did not deal with analytical procedures; nor did those that had dealt with decisions to implement such detection methods $[54,55]$ or those that had dealt with the follow up and management of detected cases [56,57]. Those that had studied biomarkers $[57,58]$ and the consensus guidelines for neonatal screening, diagnosis, and treatment $[59,60]$ as well as those that had studied costs [61] did not consider the possibility of using what has been exposed, which should improve what has been done so far, on a few diseases.
The proposed procedure, being an open method - open the window and see who's passing by; otherwise, it's usual (if he is unknown, you'll have to identify him; both the known and the unknown can be biochemical markers) - cannot establish the number of diseases to be detected. The same applies for other so called, multiplex methods. We will experiment with cases of known pathologies to control and validate the procedure.

The Gelb review [62], which refers exclusively to methods using paper dried blood spots (DBS), begins by recognizing that positive predictive value (PPV) cannot be used to evaluate programs that use enzyme activity measures. Instead, it proposes to use positive rates and biochemical markers to greatly reduce the number of FPs in the neonatal screening of lysosomal deposition diseases and suggests that these methods, after trials measuring enzyme activity are more powerful than genotyping analysis for the stratification of these diseases.

The review also refers to the use of MS/MS and Chamoles fluorometry in the measurement of enzyme activity. It mentions the use of MS/MS in the quantification of disaccharides produced by bacterial heparinidase on the glycosaminoglycan substrate in the detection of mucopolysaccharidoses, which produces many more FPs than the measurement of enzyme activity. In the case of Krabbe disease, Gelb says that FPs can be reduced if the psychosine biomarker is used in the first step (tier) compared to the measurement of enzyme activity, but measuring psychosine in DBS by MS/MS is difficult because of its low analytical sensitivity in detection, together with the need for high performance liquid chromatography (HPLC), which lasts several minutes. So, Gelb proposes it as the second tier after measuring enzyme activity as the first tier followed by an analysis of the small number of positive samples in the first tier.

He says that with similar arguments, glucosylsphingosine is used as a biomarker of Gaucher disease and in Niemann-Pick $\mathrm{A} / \mathrm{B}$, lysosphingomyelin; in this disease, he says that the biomarker elevation is modest, suggesting the existence of many FPs if the biomarker is used in the first tier. He also says that the lyso Gb3 biomarker is useful for Fabry disease, but whether this is always elevated in the neonatal period is unknown. He comments on the use of synthetic substrates, not exactly the same as natural ones, and the need for activating proteins in some cases, to the extent of enzymatic activities, which can lead to false negatives.

In our work, the advantage of neonatal screening is that which has been shown following all the steps foreseen with the initial urine and blood samples, dried on paper in this order, and the positive rate results from dividing the number of positive final results by the number of screened newborns. Finding false negatives in Galicia after looking at discharge reports and other hospital reports, which are computerized, is not difficult; we refer not only to reports of discharge from maternity after childbirth (if so, neonatal screening would not be needed) but also, to those after admission or consultation at any age.

In his review, Gelb recommends normalizing enzyme activity measures to the percentage of mean activity of the unaffected neonatal population and treats problems, interferences, and 
others that hinder this normalization. Our approach does not have the disadvantage of measuring enzyme activity. With the procedures now being used, resorting to biostatistical tools to reduce the number of FPs and, even after that, to resort to biomarkers for each disease is necessary. This is because of a clear covariation with birth weight and gestational age, so enzyme activity must be adjusted to these covariations using rigorous statistical interpolation methods. This need to resort to mathematics to establish a possible positive value tells us that we must look for other neonatal screening procedures that do not require this step. The review ends by commenting on ten lysosomal deposition diseases, for some of which no minimally developed neonatal screening program exists.

In the case of metachromatic leukodystrophy, enzyme deficiency leads to the massive accumulation of sulfated glycolipids, in particular galactosylceramide-3-O-sulphate (sulfatide). In this study, the measurement of urinary sulfatides (which do not detect "pseudo deficiency") was used, although whether neonatal screening should be considered for this disorder is questionable. Several studies exist in the literature on sulfatides and lysosulfatides. A recent study [63] employed LC-MS/MS, which is currently the "fashionable methodology,"3 and state that although plasma sulfates and lysosulfates, which could correlate with the severity of metachromatic leukodystrophy and be useful in controlling the effects of therapeutic intervention, would appear as attractive candidates for biomarkers, the results indicated that the determination of these plasma deposition compounds being useful in this regard was unlikely. Sulfatides are anionic sulfoglycolipids synthesized from their precursor galactosylceramide, which is synthesized from ceramides and uridine diphosphate galactose (UDP galactose) and is transported to the Golgi apparatus before sulfation to sulfatides.

Sulfatides consist of many molecular species with structures that differ in acyl chain length and Hydroxylation and sphingoid base. The heterogeneity of the sphingoid base composition of Sulfatides should also be considered. The accumulation of sulfates in the kidney leads to the increased excretion of sulfates in the urine, and the determination of sulfates in the urine is a convenient diagnostic tool to confirm metachromatic leukodystrophy. A January 2016 article by Spacil et al. [64] on mass spectrometry analysis for metachromatic leukodystrophy screening in dry blood and urine samples begins by defining it as a disorder caused by the deficiency of arylsulfatase A activity, which leads to the accumulation of sulfatide substrates, and states that diagnostic and monitoring procedures include the demonstration of reduced enzyme activity in peripheral blood leukocytes or the detection of sulfatides in urine. However, the study adds that developing a screening test is difficult because of enzyme instability in dried blood samples, the widespread

3 We use that expression because in most papers in this field and others, to analyze biological samples, including human ones, such a methodology is used. Our proposal to use fluorometry, which is more sensitive, transgresses this line and goes against the current. occurrence of pseudo deficient alleles, and the lack of urine samples available in neonatal screening programs.

One article [65] describes a procedure in which $3 \mathrm{~mL}$ of urine is used and arrives at the following conclusion:

Comprehensive and integrated urine screening could be very effective in the initial workup of patients suspected of having a lysosomal disorder as it covers disorders of sulfatide degradation and narrows down the differential diagnosis in patients with elevated glycosaminoglycans.

Our proposal for newborn screening uses simpler procedures with appropriate techniques.

Those used in the quoted study are hardly adaptable to a newborn screening program since they analyze the samples consecutively with prolonged analysis periods. We clearly believe that the proponents of this project point to the necessity of urine samples in neonatal screening programs.

We make the following distinctions, some consequences of not having carried out the project, and others that clarify some circumstances that could generate doubt.

The number of analyzed samples is not given as this is a research project. We cannot verify the proposal by analyzing samples as we are currently based outside the laboratory and are not in collaboration with those in charge of the laboratory, who are impeded by limited time as well as personnel and work resource cuts caused by the economic crisis and implemented policy.

There are no confirmed babies because this study has not been put into practice. Giving the percentage of positive results, suggesting the possibility of an LSD for what has already been exposed, is not possible. The follow up of positive cases does not require repeated urine or blood samples. The initial urine samples continue to be used; as expressed below, this is confirmed with blood samples received simultaneously with urine samples.

The proposal does not contemplate repeating any sample collection, when the family is informed, the disease is already confirmed. The percentage of repetition samples received is not given as no repeated sampling is applied in the proposal. FPs cannot exist since the disease is confirmed before it is communicated to the family.

Another issue is the over diagnosis of those who never developed the disease, which has already happened with PKU; because of that, early detection was not stopped. We cannot know about the false negatives since we have not put the proposal into practice. Putting the results of the application into daily practice (and into a table) should not be difficult.

The handling of both blood and urine samples obtained from and received in the laboratory does not suppose any complication given the authors' forty years of work at the Laboratory of Galicia in Santiago de Compostela [66]. Fluorometry has been introduced in many neonatal screening laboratories for the detection of LSD and other pathologies. Also, cTLC would be applied in the second step (or tier) for a few samples and is a simple and economical analytical method.

The introduction of urine samples does not imply a separate program; it avoids the described problem of the lack of sensitivity and specificity using the current procedures. 
J. inborn errors metab. screen.

The proposed procedure is simpler in its execution and more economical than current methods.

Regarding work time, the results for elevated glycosphingolipids are obtained the same day as the samples. The cTLC results are received the next day, and on the third or fourth day, those of the enzymatic study can be taken from blood samples using the procedures in programs that perform these detection methods. The genomic study could be performed the next day using the Ion Torrent.

This speculative work could be framed in what was once called natural philosophy before Kant published the Critique of Pure Reason, to be followed by what was called positivism or experimental demonstration.

The work of L.I. Woolf -received for publication on January 1,1951 - has no results and gives few clues as to how to put this proposal into practice [67].

This paper revalues urinary neonatal screening programs. What is stated here is not contemplated in the 2019 special issue of IJNS, "Newborn Screening for Lysosomal Storage Disorders: Methodologies for Measurement of Enzymatic Activities in Dried Blood Spot" [68], which compares procedures that employ enzymatic activity measurement with MS/MS with those that employ digital microfluid fluorometry (DMFF). A previous study dealt with neonatal detections that do not appear until adulthood and other aspects that provoke discussion about the suitability of these detection methods [69]. Having read the paper of May 30 [70], on the effectiveness of newborn screening methods for only four lysosomal storage disorders, it is evident that it is necessary to break with the approaches to the subject followed until now.

The implementation of the methodology and procedures proposed in this project, to incorporate them into the Newborn Screening Programs, benefits these programs and therefore, the population of newborns, to whom it is applied. The development of the project will generate the necessary evidence to justify the implementation of what is proposed in the Newborn Screening Programs. There's no previous evidence. The development of the project will contribute to improving, simplifying and reducing the cost of the Newborn Screening Programs for lysosomal storage disorders, which will be broader, more sensitive and more specific.

\section{Acknowledgments}

We thank Dr. J. R. Bonham for their support and comments.

\section{Ethics Approval and Consent to Participate}

Ethics Committee for Clinical Research of Galicia issued a favorable opinion for this research.

\section{Declaration of Conflicting Interests}

The authors declared no potential conflicts of interest with respect to the research, authorship, and/or publication of this article. In this article, the conflict lies with the current manufacturers of in vitro diagnostic materials since what is proposed is much simpler, faster, and cheaper than what has been used to date. Such a proposal will have many detractors and, should they interfere, may be doomed to failure.

\section{References}

1. Plat FM, d'Azzo A, Davidson BI, Neufeld EF, Tifft CJ. Lysosomal storage diseases. Nat Rev Dis Primers. 2018;4(1):27. doi:10.1038/s41572-018-0025-4

2. Bekri S. Laboratory diagnosis of lysosomal storage diseases. In: Mehta A, Beck M, Sunder-Plassmann G, eds. Fabry disease perspectives from 5 years of FOS. Oxford: Oxford Pharmagenesis; 2006: chapter 8. https://www.ncbi.nlm. nih.gov/books/nbk11594/

3. Wang RY, Bodamer OA, Watson MS, Wilcox WR; ACMG Work Group on Diagnostic Confirmation of Lysosomal Storage Diseases. Lysosomal storage diseases: diagnostic confirmation and management of presymptomatic individuals. Genet Med. 2011;13(5):457-484. doi:10.1097/ GIM.0b013e318211a7e1

4. Meikle PJ, Hopwood JJ, Clague AE, Carey WF. Prevalence of lysosomal storage disorders. JAMA. 1999;281(3):249254. doi:10.1001/jama.281.3.249

5. Marsden D, Levy H. Newborn Screening of Lysosomal storage disorders. Clin Chem. 2010;56(7):1071-1079. doi:10.1373/clinchem.2009.141622

6. Platt FM, Lachmann RH. Treating lysosomal storage disorders: current practice and future prospects. Biochim Biophys Acta. 2009;1793(4):737-745. doi:10.1016/j. bbamcr.2008.08.009

7. De Carvalho TG, Da Silveira Matte U, Giugliani R, Baldo G. Genome editing: Potential treatment for Lysosomal storage diseases. Curr Stem Cell Rep. 2015;1(1):9-15 doi:10.1007/ s40778-014-0007-8

8. Christensen CL, Choy FYM. A Prospective treatment option for Lysosomal storage diseases: CRISPR/Cas9 Gene editing technology for mutation vorrection in induced pluripotent dtem vells. Diseases. 2017;5(1):6. doi:10.3390// diseases5010006 $15 \mathrm{pp}$

9. Hwu WL, Chien Y-H, Lee N-C. Newborn screening for neuropathic lysosomal storage disorders. J Inherit Metab Dis. 2010;33(4):381-386. doi:10.1007/s10545-010-9130-6

10. Schielen PCJI, Kemper EA, Gelb MH. Newborn screening for Lysosomal dtorage fiseases: A concise review of the literature on dcreening methods, therapeutic possibilities and regional programs. Int J Neonatal Screen. 2017;3(2):6. doi:10.3390/ijns3020006

11. Chamoles NA, Blanco M, Gaggioli D. Diagnosis of $\alpha-\mathrm{L}-$ iduronidase deficiency in dried blood spots on filter paper: the possibility of newborn diagnosis. Clin Chem. 2001;47:780-781. doi:10.1093/clinchem/47.4.780 
12. Chamoles NA, Blanco M, Gaggioli D. Fabry disease: enzymatic diagnosis in dried blood spots on filter paper. Clin Chim Acta. 2001;308(1-2):195-196. doi:10.1016/s00098981(01)00478-8

13. Chamoles NA, Blanco M, Lorcansky S, Gaggioli D, Specola N, Casentini C. Retrospective diagnosis of GM1 gangliosidosis by use of a newborn-screening card. Clin Chem. 2001;47(11):2068. doi:10.1093/clinchem/47.11.2068

14. Parkinson-Lawrence E, Fuller M, Hopwood JJ, Meikle PJ, Brooks DA. Immunochemistry of Lysosomal storage disorders. Clin Chem. 2006;52:1660-1668. doi:10.1373/ clinchem.2005.064915

15. Meikle PJ, Ranieri E, Simonsen H, et al. Newborn Screening for Lysosomal Storage disorders: clinical evaluation of a two-tier Strategy. Pediatrics. 2004;114(4):909-916. doi:10.1542/peds.2004-0583

16. Ramsay SL, Maire I, Bindloss C, et al. Determination of oligosaccharides and glycolipids in amniotic fluid by electrospray ionisation tandem mass spectrometry: in utero indicators of lysosomal storage diseases. Mol Genet Metab. 2004;83:231-238. doi:10.1016/j.ymgme.2004.07.015

17. Spacil Z, Kumar AB, Liao H-C, et al. Sulfatide analysis by mass spectrometry for screening of metachromatic Leukodystrophy in dried blood and urine damples. Clin Chem. 2016;62(1):279-286. doi:10.1373/ clinchem.2015.245159

18. Elliott S, Buroker N, Cournoyer JJ, et al. Pilot study of newborn screening for six lysosomal storage diseases using Tandem mass spectrometry. Mol Genet Metab. 2016;118(4):304-309. doi:10.1016/j.ymgme.2016.05.015

19. Tortorelli S, Turgeon CT, Gavrilov DK, et al. Simultaneous testing for 6 Lysosomal storage disorders and $\mathrm{X}$-Adrenoleukodystrophy in dried blood spots by Tandem Mass Spectrometry. Clin Chem. 2016;62(9):1248-1254. doi:org/10.1373/clinchem.2016.256255

20. Alonso-Fernández JR, Colón C. Aportaciones de Louis I. Woolf al tratamiento y diagnóstico precoz de la fenilcetonuria $y$ otros errores congénitos del metabolismo. Los comienzos de la Tría Neonatal en España, con referencia al Programa de Galicia. 2019. doi:10.13140/rg.2.2.29898.72641

21. Alonso-Fernández JR. Tría neonatal de galactosemia: situación del ensayo en orina. Neonatal galactosaemia screening. Urine assay situation. Rev Lab Clin. 2008;1(3):133134. doi:org/10.1016/j.labcli.2008.08.001

22. Alonso-Fernández JR. Pregnanetriolone in paper-borne urine for neonatal screening for 21-hydroxylase deficiency: The place of urine in neonatal screening. Mol Genet Metab Reports. 2016;8:99-102. doi:10.1016/j.ymgmr.2016.08.006

23. Alonso-Fernández JR, Fidalgo J, Colon C. Newborn Screening for Mucopolysaccharides. Conference presented at: The 5th European ISNS Congress in Newborn Screening. June 10-12th, 2007; Reykjavik, Iceland.
24. Alonso-Fernández JR, Fidalgo J, Colon C. Neonatal screening for mucopolysaccharidoses by determining glycosaminoglycans in the eluate of urine impregnated in paper. Preliminary results of an improved DMBBased procedure. J Clin Lab Anal. 2010;24(3):149-153. doi:10.1002/jcla.20375

25. Alonso-Fernández JR, Sedes A, Colon C, Fidalgo J. Newborn Screening for Oligosaccharides. J Inherit Metab Dis. 2007;30(Suppl 1):8. doi:10.1007/s10545-010-9163-X

26. Alonso-Fernández JR, Carpinteiro MI, Baleato J, Fidalgo J. Continuous evaporative TLC with Small eluent volumes for saccharides and oligosaccharides of clinical interest. Paper presented at: VI Reunión Científica de la SECyTA. November 8-10th, 2006; Vigo, Spain.

27. Alonso-Fernández JR, Carpinteiro MI, Baleato J, Fidalgo J. Vertical Sandwich-Type continuous/evaporative TLC with fixed mobile phase volume for separating sugars of vlinical relevance in paper-borne urine and blood samples in newborn screening. J Clin Lab Anal. 2010;24(2):106-112. doi:10.1002/jcla.20371

28. Alonso-Fernández JR, Patel VB. Dietary sugars: TLC screening of sugars in urine and blood samples. In: Preedy VR. Dietary sugars: Chemistry, analysis, function and effects. London: The Royal Society of Chemistry; 2012: 186-207. doi:10.1039/9781849734929-00186

29. Fidalgo López J, Alonso-Fernández JR. Peneirado neonatal das enfermidades de depósito lisosomal empregando ouriños enxoitados en papel: o rexurdimento das mostras de ouriños para o diagnóstico das glicosfingolipidoses. Conference presented at: Xornada de divulgación científica Primeiros pasos na ciencia. October 7th, 2016; Lugo, Spain. doi:10.13140/RG.2.2.26566.68160

30. Alonso-Fernández JR, Bóveda MD, Parrado C, Peña J, Fraga JM. Continuous thin-layer chromatography of sugars of clinical interest in samples of urine impregnated on paper. J Chromatogr. 1981;217:357-366. doi:10.1016/s00219673(00)94856-8

31. Young Jr WW, Borgman CA. Short bed-continuous development thin-layer chromatography of glycosphingolipids. J Lipid Res. 1986;27:120-124. doi:10.1016/S0022-2275(20)38862-3

32. Auray-Blais C, Cyr D, Mills K, Giguere R, Drouin R. Development of a filter paper method potentially applicable to mass and high-risk urinary screening for Fabry disease. J Inherit Metab Dis. 2007;30(1):106. doi:10.1007/s10545006-0444-3

33. Watanabe K, Mizuta M. Fluorometric detection of glycosphingolipids on thin-layer chromatographic plates. J Lipid Res. 1995;36(8):1848-1855. doi:10.1016/S00222275(20)41503-2

34. Actualización do programa galego para a detección precoz de enfermedades endócrinas e metabólicas en periodo neonatal. 
Resultados 1995-2006. Xunta de Galicia. Conselleria de Sanidade. Dirección Xeral de Saúde Pública, 2008. https:// dialnet.unirioja.es/descarga/libro/516184.pdf

35. Moskot M, Bochenska K, Jakóbkiewicz-Bancka J, Banecki B, Gabig-Ciminska M. Abnormal sphingolipid world in inflamation specific for Lysosomal storage diseases and skin disorders. Int J Mol Sci. 2018;19:247. doi:10.3390/ ijms 19010247

36. Boutin M, Menkovic I, Martineau T, VaillancourtLavigueur V, Toupin A, Auray-Blais C. Separation and analysis of lactosylceramide, galabiosylceramide and globotriaosylceramide by LC-MS/MS in urine of Fabry disease patients. Anal Chem. 2017;89(24):13382-13390. doi:10.1021/acs.analchem.7b03609

37. Gnirke A, Melnikov A, Maguire J, et al. Solution hybrid Selection with ultra-long oligonucleotides for massively parallel targeted sequencing. Nat Biotechnol. 2009;27:182189. doi:10.1038/nbt.1523

38. Fernández-Marmiesse A, Morey M, Pineda $\mathrm{M}$, et al. Assessment of a targeted resequencing assay as a support tool in the diagnosis of lysosomal storage disorders. Orpha J Rare Dis. 2014;9:59. doi:10.1186/1750-1172-9-59

39. Wald NI, Bestwick J. Is the area under an ROC curve a valid measure of the performance of a screening or diagnostic test? J Med Screen. 2014;21(1):51-56. doi:10.1177/0969141313517497

40. Millington DS, Bali DM. Misinformation regarding tandem mass spectrometric vs fluorometric assays to screen newborns for LSDs. Mol Genet Metab Reports. 2017;11:7273. doi:10.1016/j.ymgmr

41. Gelb MH, Scott CR, Turecek F, Liao H-C. Comparison of tandem mass spectrometry to fluorimetry for newborn screening of LSDs. Mol Genet Metab Reports. 2017;12:8081. doi:10.1016/j.ymgmr

42. Gelb MH, Ronald Scott C, Turecek F, Liao HC. Comparison of tandem mass spectrometry to fluorimetry for newborn screening of LSDs. Mol Genet Metab Reports. 2017;12:8091. doi:10.1016/j.ymgmr

43. Millington DS, Bali DS. Current state of the art of newborn screening for Lysosomal storage disorders. Int J Neonatal Screen. 2018;4(3):24. doi:10.3390/ijns4030024

44. Lu Y-H, Huang P-H, Wang L-Y, et al. Improvement in the sensitivity of newborn screening for Fabry disease among females through the use of a high-throughput and costeffective method, DNA mass spectrometry. J Hum Genet. 2018;63:1-8. doi:10.1038/s10038-017-0366-y

45. Menéndez-Sainz MC, González-Garcia S, GonzálezQuevedo A, et al. A proposal to consider: sensitivity, specificity and normal range values of enzymatic deficiency of lysosomal enzymes. SCIREA J Clin Med. 2016;1(2):323-335. http://www.scirea.org/journal/ paperinformation? paperid $=241$
46. Eyskens F, Devos S. Newborn screening for Lysosomal storage disorders in Belgium: The importance of sex and age-dependent reference ranges. J Inborn Errors Metab Screen. 2017;5:1-8. doi:10.1177/2326409817744231

47. La Marca G. Lysosomals. In: Blau N, Duran M, Gibson $\mathrm{K}$, Dionisi Vici C, eds. Physician's guide to the fiagnosis, treatment, and follow-up of inherited metabolic diseases. Berlin, Heidelberg: Springer; 2014, doi:10.1007/978-3642-40337-8_52

48. Supriya M, De T, Christofer R. Effect of temperature on Lysosomal enzyme activity during preparation and storage of dried spots. J Clin Lab Anal. 2017; 32(1):e22220. doi:10.1002/jcla.22220

49. Alonso-Fernández JR, Graña I, Iglesias AJ. Screening for Familial Hypercholesterolaemia by Immunoturbidimetric Quantitation of Apolipoproteins A-I and B in dried blood spot. Paper presented at: 8th International Neonatal Screening Symposium and Inaugural Meeting. International Society for Neonatal Screening, Australia; 1991.

50. Moat SJ, Korpimäki T, Furu P, Hakala H, Polari H, Meriö L, Mäkinen P, Weeks I. Characterization of a blood spot creatine kinase skeletal muscle isoform immunoassay for High-Throughput newborn screening of Duchenne Muscular Dystrophy. Clin Chemm. 2017;63(4):908-914. doi:10.1373/clinchem.2016.268425

51. Ferreira CR, Gahl WA. Lysosomal storage diseases. Transl Sci Rare Dis. 2017;2(1-2):1-71. doi:10.3233/trd-160005

52. Anderson S. Newborn Screening for Lysosomal Storage Disorders. J Pediatr Health Care. 2018;32(3):285-294. doi:10.1016/j.pedhc.2017.04.016

53. Bellettato CM, Hubert L, Scarpa M, Wangler MF. Inborn errors of metabolism involving complex molecules. Lysosomal and Peroxisomal storage diseases. Pediatr Clin N Am. 2018;65(2):353-373. doi:10.1016/j.pcl.2017.11.011

54. Prosser LA, Lam KK, Grosse SD, Casale M, Kemper AR. Using decision analysis to support newborn screening policy decision: A case study for Pompe disease. MDM Policy \& Practice 2018;3(1):2381468318763814. doi:10.1177/2381468318763814

55. Langeveld M. Editorial commentary: Newborn screening for Fabry disease: Too much too soon? Trends in Cardiovasc Med. 2018;28(4):282-283. doi:10.1016/j.tcm.2017.12.015

56. Franzen D, Haile SR, Kasper DC, Mechtler TP, Flammer AJ, Krayenbühl PA, Nowak A. Pulmonary involvement in Fabry disease: effect of plasma globotriaosylsphingosine and time to initiation of enzyme replacement therapy. BMJ Open Resp Res. 2018;5:e000277. doi:10.1136/bmjresp-2018-000277

57. Pasqualim G, dos Santos BA, Giugliani R, Matte U. Simple and efficient screening of patients with Fabry disease with high resolution melting. Clin Biochem. 2018;53:160-163. doi:10.1016/j.clinbiochem.2018.01.002 
58. Alharbi FJ, Baig S, Auray-Blais C, et al. Globotriaosylsphingosine (Lyso-Gb3) as a biomarker for cardiac variant (N2155) Fabry disease. J Inherit Metab Dis. 2018;41(2):239-247. doi:10.1007/s10545-017-0127-2

59. Kwon JM, Matern D, Kurtzberg J, et al. Consensus guidelines for newborn screening, diagnosis and treatment of infantile Krabbe disease. Orphanet J Rare Dis. 2018;13(1):30. doi:10.1186/s13023-018-0766-x

60. Geberhiwot T, Moro A, Dardis A, et al. Consensus clinical management guidelines for Niemann-Pick disease type C. Orphanet J Rare Dis. 2018;13(1):50. doi:10.1186/s13023018-0785-7

61. Guest JF, Ingram A, Ayoub N, et al. Healthcare resource use and cost of managing children and adults with lysosomal acid lipase deficiency at a tertiary referral center in the United Kingdom. PloS ONE. 2018;13(2):e0191945. doi:10.1371/journal.pone.0191945

62. Gelb MH. Newborn Screening for Lysosomal Storage Diseases: Methodologies, screen positive rates, normalization of datasets, Second-Tier Tests, and post-analysis tools. Int J Neonatal Screen. 2018;4(3):23. doi:10.3390/ ijns4030023

63. Mirzaiian M, Kramer G, Poorthuis BJHM. Quantification of sulfatides and lysosulfatides in tissues and body fluids by liquid chromatography-tandem mass spectrometry. $J$ Lipid Res. 2015;56(4):936-943. doi:10.1194/jlr.m057232
64. Spacil Z, Kumar AB, Liao H-C, et al. Sulfatide analysis by mass spectrometry for screening of metachromatic Leukodystrophy in dried blood and urine samples. Clin Chem. 2016;62(1):279-286. doi:10.1373/ clinchem.2015.245159

65. Pino G, Conboy E, Tortorelli S, et al. Multiplex testing for the screening of lysosomal storage disease in urine: Sulfatides and glycosaminoglycan profiles in 40 cases of sulfatiduria. Mol Genet Metab. 2020;129(2):106-110. doi:10.1016/j.ymgme.2019.10.009

66. Couce ML. Fifty years of neonatal screening for congenital diseases in Spain. An Pediatr (Barc). 2019;90(4):205-206. doi:10.1016/j.anpedi.2018.11.013

67. Woolf LI, Vulliamy DG. Phenylketonuria with a study of the effect upon it of Glutamic Acid. Arch Dis Child. 1951;26(130):487-94. doi:10.1136/adc.26.130.487

68. Gelb MH, Lukacs Z, Ranieri E, Schielen P. Newborn screening for lysosomal storage disorders: methodologies for measurement of enzymatic activities in dried blood spots. Int J Neonatal Screen. 2019;5(1):1. doi:10.3390/ ijns5010001

69. Wilcken B. Newborn screening for Lysosomal disease: Mission creep and a taste of things to come? Int J Neonatal Screen. 2018;4(3):21. doi:10.3390/ijns4030021

70. Sanders KA, Gavrilov DK, Oglesbee D, et al. A Comparative effectiveness study of newborn screening methods for four Lysosomal storage disorders. Int J Neonatal Screen. 2020;6(2):44. doi:10.3390/ijns6020044 